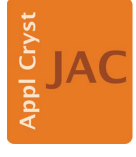

JOURNAL OF APPLIED CRYSTALLOGRAPHY

ISSN 1600-5767

Keywords: book reviews

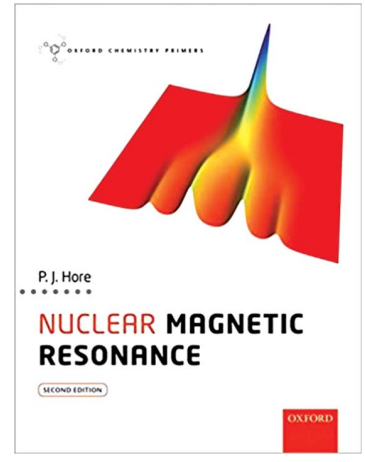

(C) 2017 International Union of Crystallography

\section{Nuclear Magnetic Resonance. Second Edition. By Peter Hore. Oxford University Press, 2015. Pp. 120. Price GBP 14.99. ISBN 9780198703419.}

\author{
Sabine Bouguet-Bonnet* \\ Université de Lorraine, CRM2, UMR 7036, Vandoeuvre-les-Nancy, F-54506, France, and CNRS, CRM2, UMR 7036, \\ Vandoeuvre-les-Nancy, F-54506, France. *Correspondence e-mail: sabine.bonnet@univ-lorraine.fr
}

This book is indisputably a 'must have' for any student, or even teacher, in the field of nuclear magnetic resonance (NMR). It is the second edition of Nuclear Magnetic Resonance by P. J. Hore, a reference book for NMR in the liquid phase. The book is mainly intended for students in chemistry who wish to understand the physical bases of NMR spectroscopy: the text is written in a concise and very clear way, and all the necessary notions are explained in only 112 pages. It is a perfect format for preparing readers for more advanced studies on the topic. Theoretical explanations are illustrated by many examples and applications all through the book, and this second edition has been completed with a series of exercises the solutions of which are provided at the end of the book. More details of these solutions are available online via the publisher's web site.

The text is divided into six chapters, each one ending with a short summary and a series of exactly ten exercises. Following an appendix about magnetic dipoles, there is a glossary that would be very interesting for those unfamiliar with NMR, and then a short bibliography, followed by the solutions (in a short version) to the exercises.

Chapter 1 is a very good introduction to the topic. Here are introduced the interactions that will be detailed and explained in the following chapters. Chapter 2 is dedicated to chemical shifts. Unlike the first edition, clarifications on the sign of the Larmor frequency are given here. The origins of chemical shifts are covered in great detail: the different contributions are mentioned and clearly illustrated by many examples. This part is definitely going to attract much interest from readers with a background in chemistry. The following chapter is logically dedicated to spin-spin coupling. Here again, many examples allow the reader to fully assimilate the theory. At the end of this chapter, a section covers dipolar coupling. After the chapters concerning the interactions giving rise to NMR spectra in solution (chemical shifts and $J$ couplings), the following chapters are dedicated to dynamic processes, namely exchange (Chapter 4) and spin relaxation (Chapter 5), and their effect on the appearance of NMR lines. Always, for the sake of simplicity, P. J. Hore succeeds in presenting NMR relaxation without the formal use of Bloch's equations. The section concerning motion, and its link to the relaxation, is however addressed quickly and would merit more explanation. In particular, the Lorentzian form of the spectral density arrives at the reader a little from nowhere. Similarly, the presentation of the nuclear Overhauser effect is rather muddled without the Solomon equations. Even so, this chapter about spin relaxation is pleasant to read and very informative, thanks to its long list of examples and applications which have been clearly separated into two subsections corresponding to structural and dynamic. The sixth and last chapter covers practical NMR and the manipulation of spins. As indicated by the author in his preface, this chapter is a foretaste for the reader willing to go deeper into NMR pulse sequences and the underlying quantum mechanics, topics which are developed in another Oxford Chemistry Primer from the same author (NMR: The Toolkit). Welcome additions have been made compared to the first edition in this chapter. They concern INEPT (insensitive nuclei enhanced by polarization transfer), HSQC (heteronuclear single quantum correlation) and a short section about three-dimensional NMR.

To conclude, this primer fully deserves to be widely adopted by students (and teachers) of NMR spectroscopy. I think this book is a complete and very accessible tool for understanding the origin and significance of the basic NMR parameters. 